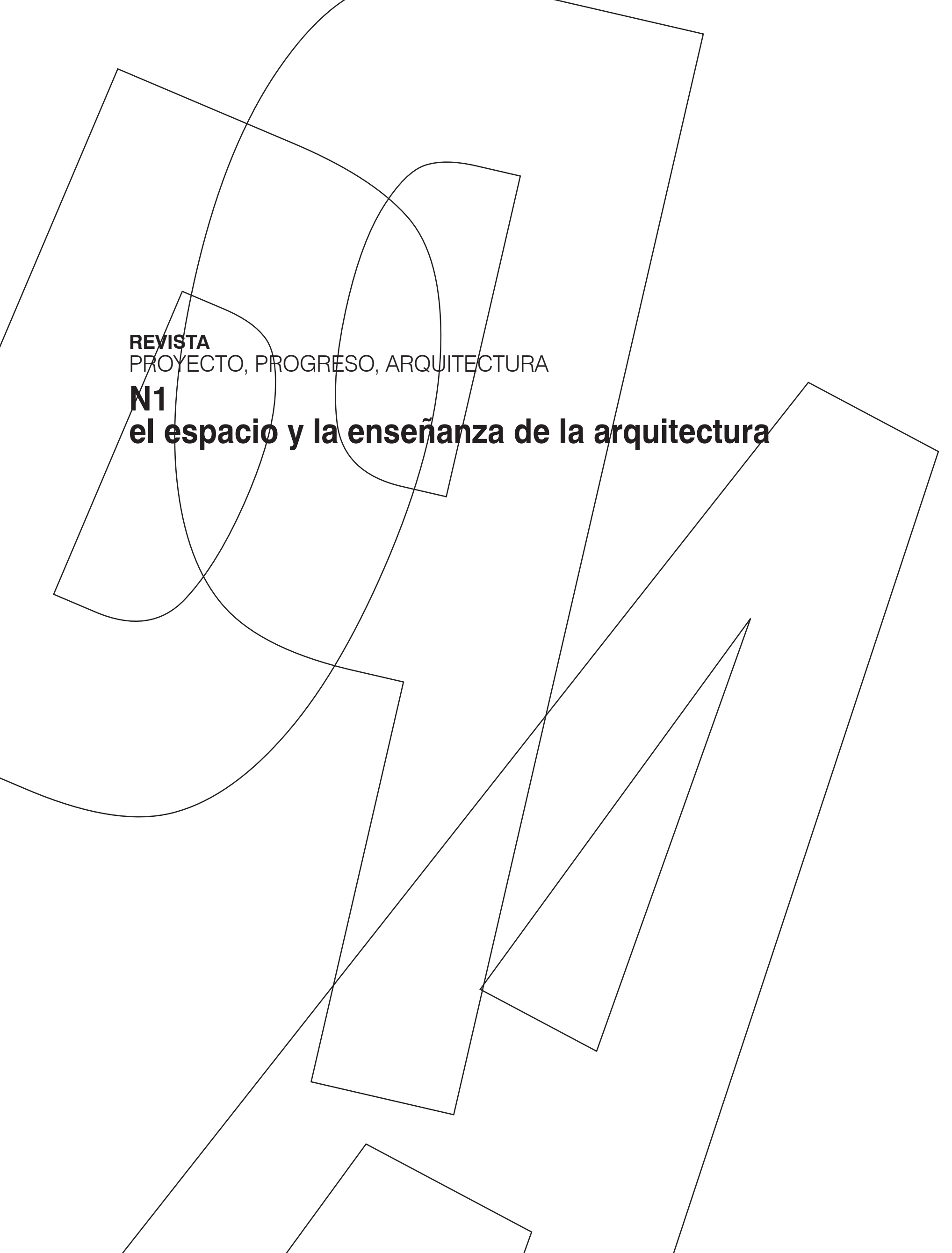


RESUMEN El edificio de la Escuela Técnica Superior de Arquitectura de Valencia pertenece al proyecto unitario de las cuatro escuelas que en el año 1970 fue ganado por el equipo L35. El realismo económico y técnico, así como la adopción de los postulados de la carta de Atenas, sintetizados para la ciudad funcional, tienen como resultado la separación de la ciudad en cuatro áreas funcionales: trabajo, residencia, esparcimiento y circulación. Dentro de esta disposición, se asigna a la universidad la zona destinada al trabajo, que se concreta en la docencia y la investigación.

Las técnicas de construcción no han cambiado sustancialmente desde el proyecto inicial, pero tras 40 años ya se han podido experimentar y valorar los resultados de la prefabricación. El deterioro debido a las inclemencias del tiempo apoya la elección de una técnica más afín con los resultados de una mayor durabilidad, llevando la cantería a un grado de fabricación que dentro del procedimiento manufacturero es tan actual como adaptable. Las técnicas actuales posibilitan la traducción de los fabricados industrializados a un código arquitectónico donde encuentran su lógica expresiva. Se interpreta el fabricado de la piedra de la piedra actualizando su código constructivo para obtener una expresividad manual y visual que los prefabricados no pueden ofrecer.

PALABRAS CLAVE ley, regla, jardín, fabricado, pasarela, taller.

SUMMARY The building of the Higher Technical School of Architecture of Valencia pertains to the joint project of the four schools, which was won by the L35 team in 1970. Economic and technical realism, as well as the adoption of the postulates of the Athens Charter, synthesized for the functional city, had the result of separating the city into four functional areas: living, working, recreation and circulation. Within this arrangement, the university is assigned to the area destined for teaching and research, the urban space destined for working.

The construction techniques had not changed substantially from the initial plan, but after 40 years it has been possible to experience and evaluate the results of prefabrication. The observation that some heavy panels were superficially deteriorated by inclemencies of time, led us to choose a technique more compatible with the results that we hoped to obtain. This took stone working to a level of manufacture that is both current and adaptable in the production procedure. The present techniques enable the translation of industrialised products into an architectural code where they find their expressive logic. We have interpreted stone technology, updating its constructive code, to obtain a manual expressivity that the prefabricated material cannot offer

KEY WORDS law, rule, garden, manufactured, balcony walkway, workshop. 


\section{LA PUESTA AL DÍA DE UNA ESCUELA DE ARQUITECTURA}

UPDATING A SCHOOL OF ARCHITECTURE

Vicente Manuel Vidal Vidal, Ciro Manuel Vidal Climent

\section{ANTECEDENTES}

n el año 1970 se convoca el concurso conocido como "Segunda Fase del Politécnico" con la pretensión de configurarse en el futuro como el gran centro nacional de investigación técnica aplicada. Allí se impartirían enseñanzas de grado medio y superior de las ramas de Arquitectura, Ingeniería de Caminos, Industriales, y Agrónomos.

Se presentaron 13 trabajos al concurso, siendo el equipo catalán L35 el que obtuvo el primer premio. El proyecto presentado se basaba en la segregación de un sistema de circulación rodada, a nivel $+0,00$ y el acceso peatonal a la cota $+4,00$. La base de la trama original que ordenaba la construcción del conjunto adoptaba una trama geométrica de 2,5 m con un submúltiplo de 1,25 m y múltiplos de 5 y 7,50 m que eran a su vez, la base de la trama estructural. La estructura estaba prevista como un sistema de pilares y jácenas prefabricados de hormigón, compuestos en pórticos ensamblables en obra mediante el hormigonado de los nudos.

En el año 1999, el rectorado de la Universidad Politécnica de Valencia decidió incorporar la experiencia de los profesores de proyectos a las obras que la universidad realizaba en su ampliación y renovación. Para ello se convocaron tres concursos consecutivos.

Un primer concurso para la eliminación de las plataformas de cota $+4,00$ que conectaban entre sí las cuatro escuelas originarias (Agrónomos, Industriales, Caminos y Arquitectura) con el Rectorado.

Un concurso para la sede del Vicerrectorado de Cultura, que no se llevó a cabo.

Otro concurso para la remodelación y ampliación de la Escuela Técnica Superior de Arquitectura, que se falló a favor del proyecto que presentamos en este artículo.

La propuesta original de las cuatro escuelas, correspondiente al concurso de 1970, basándose en la idea de la producción en serie y la prefabricación integral, se transformó en fase de proyecto y construcción a través de dos cambios:

El aumento del módulo original, adoptando el nuevo módulo de $3 \mathrm{~m}$, con un submúltiplo de 1,5 m y múltiplos de 6 y $9 \mathrm{~m}$
La adopción en su construcción de un sistema mixto de estructura de pilares y jácenas de hormigón in situ, de 6 y $9 \mathrm{~m}$ de luz, y de forjados de losas aligeradas prefabricadas de $6 \mathrm{~m}$ de luz.

La planta isótropa resultante es inicialmente una cuadrícula de $6 \mathrm{~m}$, con un submúltiplo modular de 1,5m que dimensionaba cielos rasos, iluminación, aireación y claraboyas cenitales. Estas claraboyas eran los puntos débiles que imposibilitaban la atemperación de las salas a causa de su excesiva radiación solar.

La planta estaba ordenada a la manera de un cardo en dirección Norte-Sur y un decumano Este-Oeste, con la anomalía de que la circulación Este-Oeste, que se configuró como principal, mantenía una sección la mitad de ancha que la Norte-Sur.

Todas las instalaciones de atemperación estaban obsoletas y la cerrajería y la carpintería eran de una calidad prescindible.

\section{SOBRE EL PROYECTO}

\section{Fases}

Para no interrumpir el funcionamiento docente el proyecto se pensó en tres fases, cuya ejecución dejaba siempre la suficiente cantidad de aulas libres para poder desarrollar la docencia.

La primera fase se centró en el área Sur-Oeste, ampliándose toda la cota $+8,00$ con nuevas dotaciones que consistían en 6 salas de proyectos, asistidas por 6 aulas teóricas y los despachos que corresponden al departamento de proyectos. En la cota $+4,00$ las 6 antiguas aulas de proyectos con iluminación cenital se transforman en 12 aulas con iluminación lateral continua.

En la segunda fase se actuó en el sector Sur-Este, que corresponde a la zona de dirección y secretaría de la escuela, los despachos y talleres de los departamentos de Construcción, Materiales, Mecánica del suelo, Instalaciones, Estructuras, Urbanismo y Matemática aplicada. En esta zona se instaló también el Centro de Información Arquitectónica (CIA). En la planta baja de este sector se recupera el espacio antiguamente cedido a los coches, recobrando para las escuelas de 

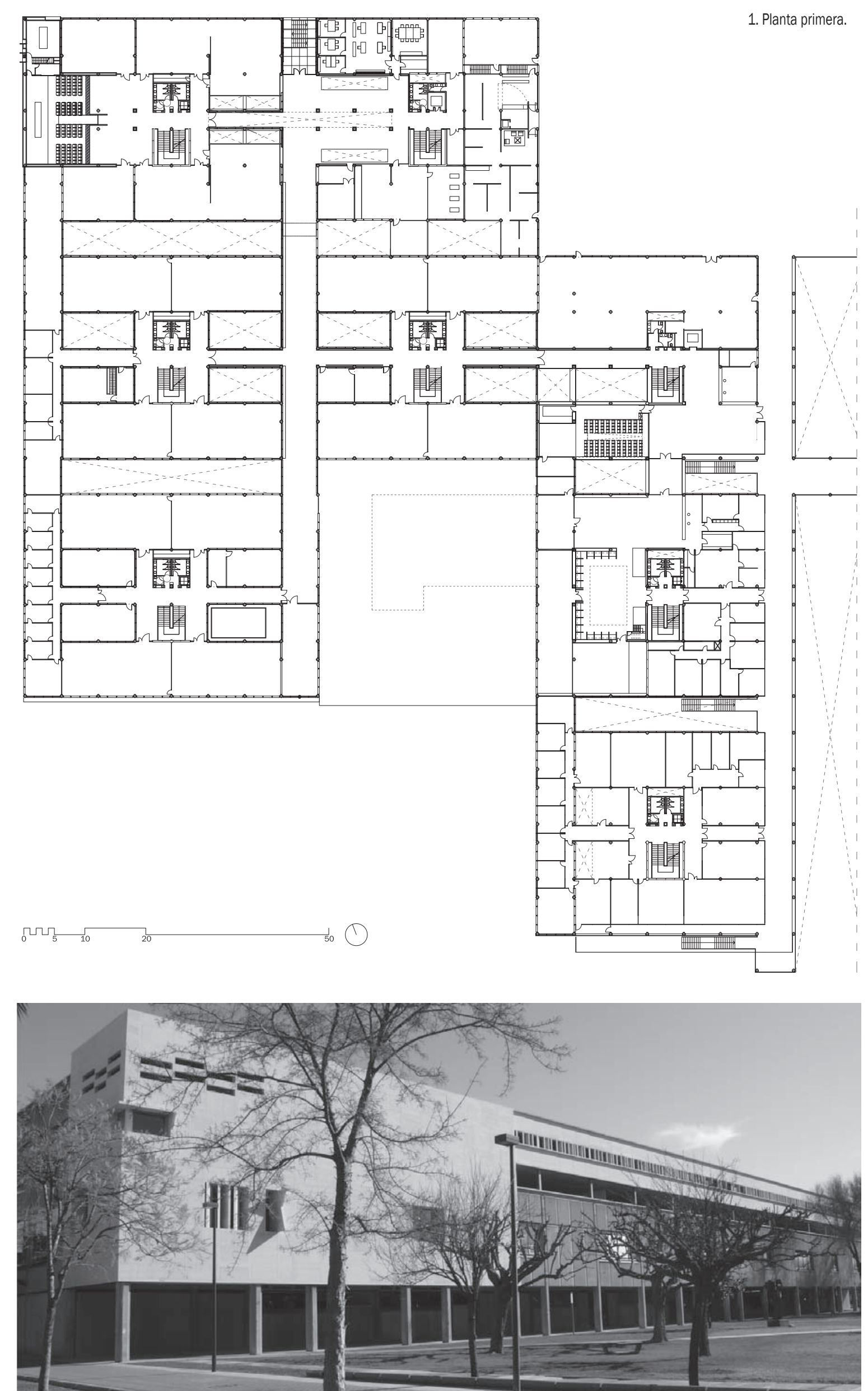
arquitectura y de caminos un espacio amplio de aulas y una calle ajardinada entre ellas.

La tercera fase consistió en la construcción del Aula Magna y la remodelación de la zona Noroeste, donde se sitúa la secretaría del departamento de Proyectos Arquitectónicos y los despachos del departamento de Expresión Gráfica.

\section{Ley de composición interna}

Los principios del concurso origen de las cuatro escuelas estaban inspirados en un realismo económico y técnico sobre la flexibilidad del conjunto y de sus elementos, orientados a conseguir una solución abierta a toda transformación futura. Estos principios implicaban una eliminación de matices donde la forma se subordinaba al módulo, oponiéndose al caos y llevando las soluciones a la lógica y la precisión modular.

La transformación de la Escuela de Arquitectura implica atender a la ley de composición del proyecto original, proponiendo las reglas que mantengan la coherencia entre el proyecto construido en los años 70 y la remodelación necesaria para la puesta al día de la Escuela.

\section{Reglas de proyecto}

Es necesario establecer las reglas que nos permitan actuar con la lógica interna de la construcción heredada y transformarla a tenor de las nuevas exigencias utilizando un código arquitectónico preciso.

Estas reglas son necesarias para entender y poder explicar la nueva disposición a partir de la construcción anterior. Su enumeración aclarará la lectura del proyecto y éstas son:

1. La aceptación de la linealidad de las circulaciones principales en sentido Este-Oeste y su desarrollo espacial, tanto horizontalmente a través de las enfiladas Este-Oeste que terminan en vistas hacia los jardines del campus, como verticalmente a través de la relación visual entre la planta de servicio a cota $+4,00$ y la superior.

2. Rescatar de las cesuras del proyecto original una estructura ya ausente de patios, con el fin de reintroducir la luz en los distintos niveles que actualmente carecen de ella, produciendo una atmósfera basada en la luz filtrada a través de la transformación de estos patios en jardines.
3. Asumir la envolvente de los prefabricados existentes de fachada para no perder la homogeneidad del conjunto formado por las cuatro escuelas. Esta cualidad pétrea existente nos aconseja introducir nuevos paramentos de piedra tersos y lisos con el objetivo de ser fieles a la luz reflejada y no a las texturas preexistentes

4. Mostrar la transición entre la masa discontinua del prefabricado y los nuevos volúmenes aristados y continuos de piedra, a través de la separación con masas de vidrio de gran fricción o vacíos de aire y sombra.

5. Hacer perceptible internamente la estructura vertical asociada a escaleras, bloques de aseos y ascensores.

6. Emplear un armazón ligero de acero sobre la estructura pesada de hormigón eliminando la presencia reiterativa de los pilares de hormigón.

Estos criterios forman el armazón teórico que facilita las decisiones de proyecto siguientes:

\section{Puesta en valor de las circulaciones}

Las leyes de composición del edificio isótropo existente no resultan coherentes con los sistemas lineales de circulación y el orden vertical de las escaleras, servicios y aulas. La regla que nos permitió reordenar la escuela fue la aceptación de la linealidad de las circulaciones al eliminar la ortotropía de los antiguos falsos techos (figura 1). No obstante éramos conscientes que la utilización obsesiva del módulo resulta tan banal como la dirección única si no existe voluntad compositiva.

Se extiende a todo el edificio la lectura lineal EsteOeste. Los pasillos se dinamizan a través de la iluminación lateral, con paramentos transparentes de gran medida para la luz fría y uniforme de Norte y con vidrios de menor altura para la luz cálida e intensa del Sur.

La sección de los pasillos Este-Oeste se duplica en altura, afirmando así el valor jerárquico de la circulación principal de cota $+4,00$ que se asume como planta de servicios a partir de la cual se distribuye en altura el resto de programa del edificio (figura 2). La planta baja queda así en gran parte liberada y dedicada a laboratorios y talleres, así como un nuevo acceso en el Este a cota $+0,00$ vinculado a la sala de exposiciones temporales y las oficinas de intercambio internacional. 

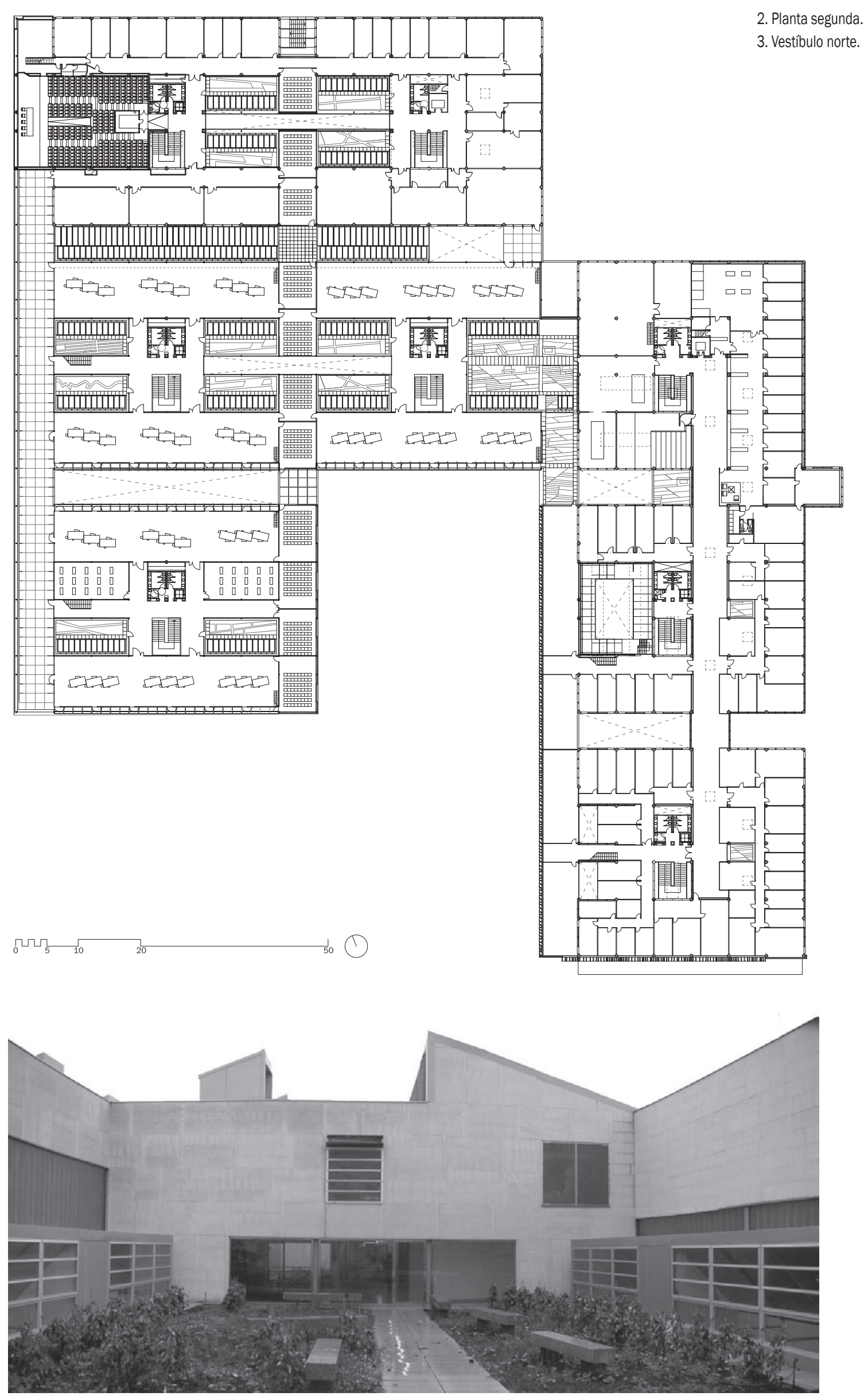

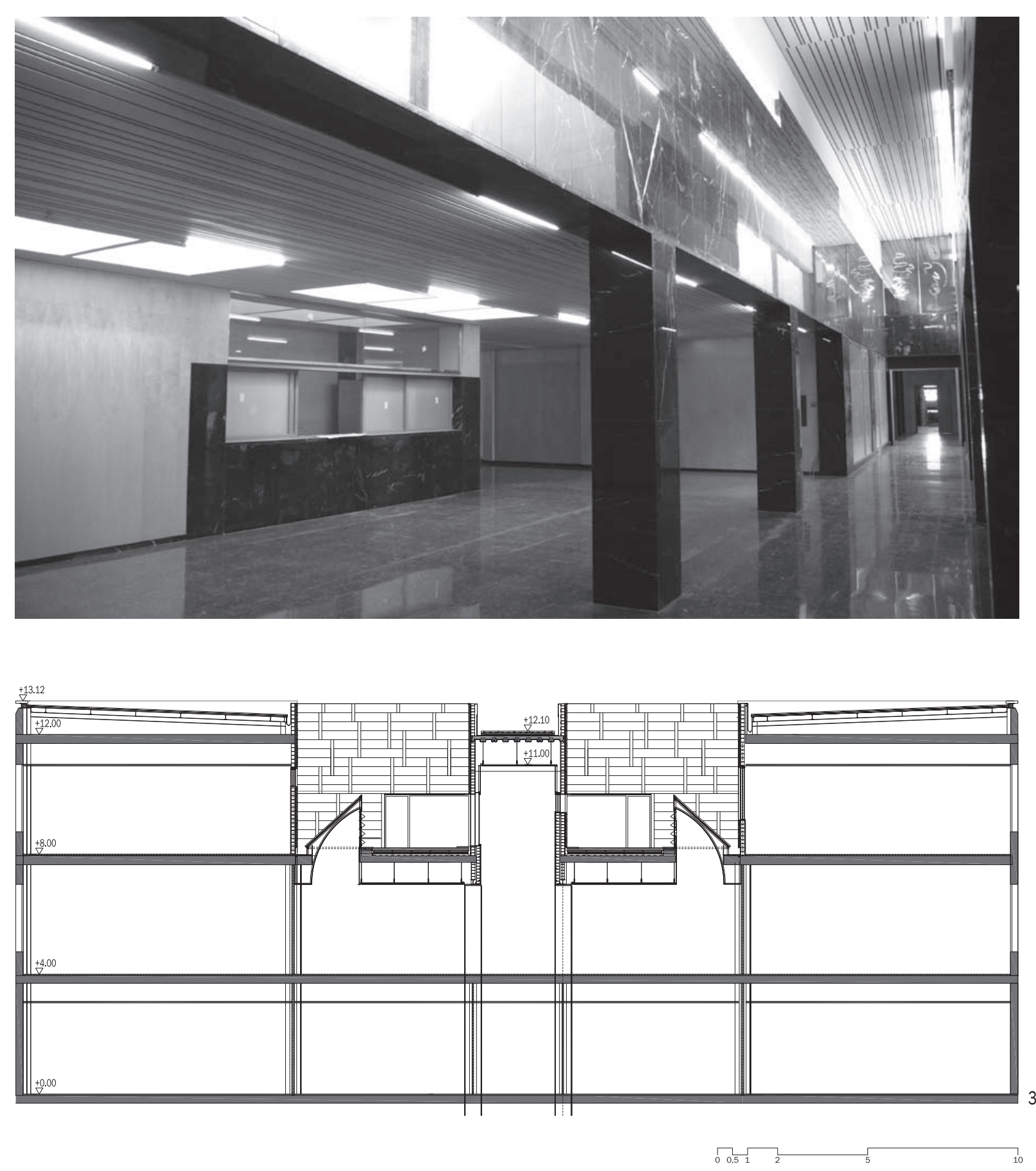

Los vestíbulos de acceso

Hay dos vestíbulos de acceso en la cota $+4,00$, uno vinculado a la puerta Este y el otro situado después de subir la escalera Norte desde el jardín del campus.

Desde el hall Este, se accede a las Salas de Grados, al Centro de Información y Documentación Arquitectónica, a Secretaría y Dirección, y al pasillo principal Este-Oeste.

El vestíbulo Norte, de mayor dimensión, es un espacio hipóstilo (figura 3), de doble altura y con una fuerte marca de luz reflejada sobre negro marquina nos acompaña un trazo recortado en el vinilo hasta las aulas de examen y el Aula Magna.
Accedemos al hall Norte a través de una escalera másica, que forma parte de la composición de fachada y se vincula directamente al jardín. Está formada por tres tramos que se van retirando en planos según se asciende (figura 4).

Los patios como elementos vertebradores del proyecto En la planta $+8,00$ aparecen los patios de $6 \times 12$ m como jardines interiores de plantación vegetal autóctona. Orientados en la dirección Este-Oeste estos patios iluminan tanto la planta a la que pertenecen como los pasillos de 
4. Escalera norte.

5. Enfilada oeste.

6. Galería sur.

7. Centro de Información Arquitectónica.

cota $+4,00$ a través de la doble altura de los mismos. Esta disposición introduce una vinculación visual entre las dos plantas mediante vistas cruzadas.

Estos patios ajardinados introducen orden y claridad en los rellanos de espera en la cota $+8,00$ y permiten conexiones visuales que atraviesan los espacios de escaleras y recorridos entre las aulas y la terraza de poniente. (figura 5).

\section{Aulas de proyectos}

La aulas de proyectos tienen una dimensión de 9x45m y $4,7 \mathrm{~m}$ de altura. Una pasarela lateral utilizada como espacio expositivo -disposición que fue tomada por las academias de dibujo del siglo XIX- las recorre en toda su longitud a la manera de los antiguos talleres manufactureros que conectaban el taller con las salas técnicas, como se muestra en el Edificio de Investigación de Minerales y Metales del IIT.

A través de este recorrido a 2,20 m por encima del plano del aula se provoca una sombra en el sur al retirar $1,20 \mathrm{~m}$ el plano de vidrio al interior del aula a la vez que se

4

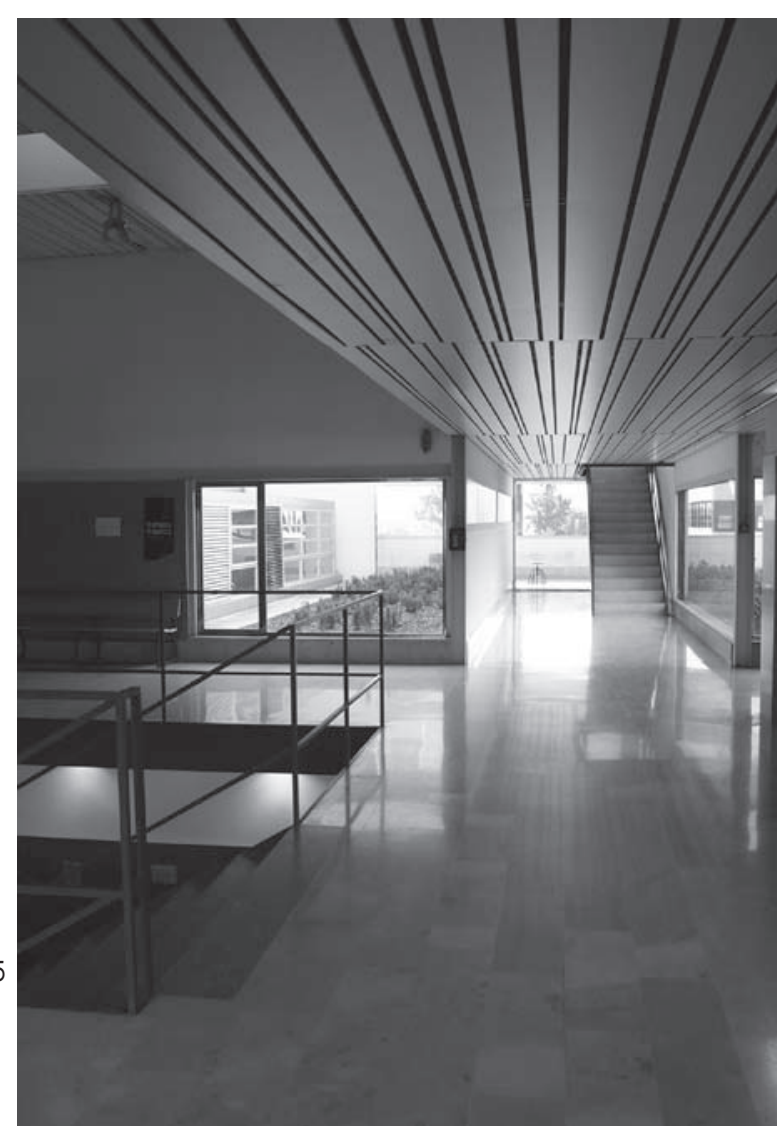
generan galerías exteriores que protegen de la insolación el vidrio y facilitan su limpieza (figura 6). Esta "promenade" discurre entre las aulas de teoría situadas en el frente Este y los despachos del departamento de proyectos, situados sobre la terraza Oeste. Las aulas disponen de esta terraza en su misma cota, que las dilata visual y funcionalmente, al poder utilizarse como espacio docente en primavera, pues está volcada al jardín del campus donde se observa el desarrollo de la vegetación.

Las salas de proyectos mantienen una disposición lineal Este-Oeste, iluminadas lateralmente con las vistas protegidas del Sur y en la pared opuesta por una alta luz norte reforzada con las lumbreras de techo que al mismo tiempo sirven para ventilar el aula.

\section{El Centro de Información Arquitectónica}

La sala principal está organizada como un gran vacío central con iluminación natural en la parte superior a través de dos lucernarios lineales. Esta pieza tiene una altura libre de $8 \mathrm{~m}$. En el espacio de la sala se desarrolla un recorrido perimetral escalonado, que va ascendiendo y creando cubículos de trabajo. A lo largo de todo el recorrido se sitúan las estanterías de los libros de consulta. En torno a esta gran sala central se organizan el resto de salas de 


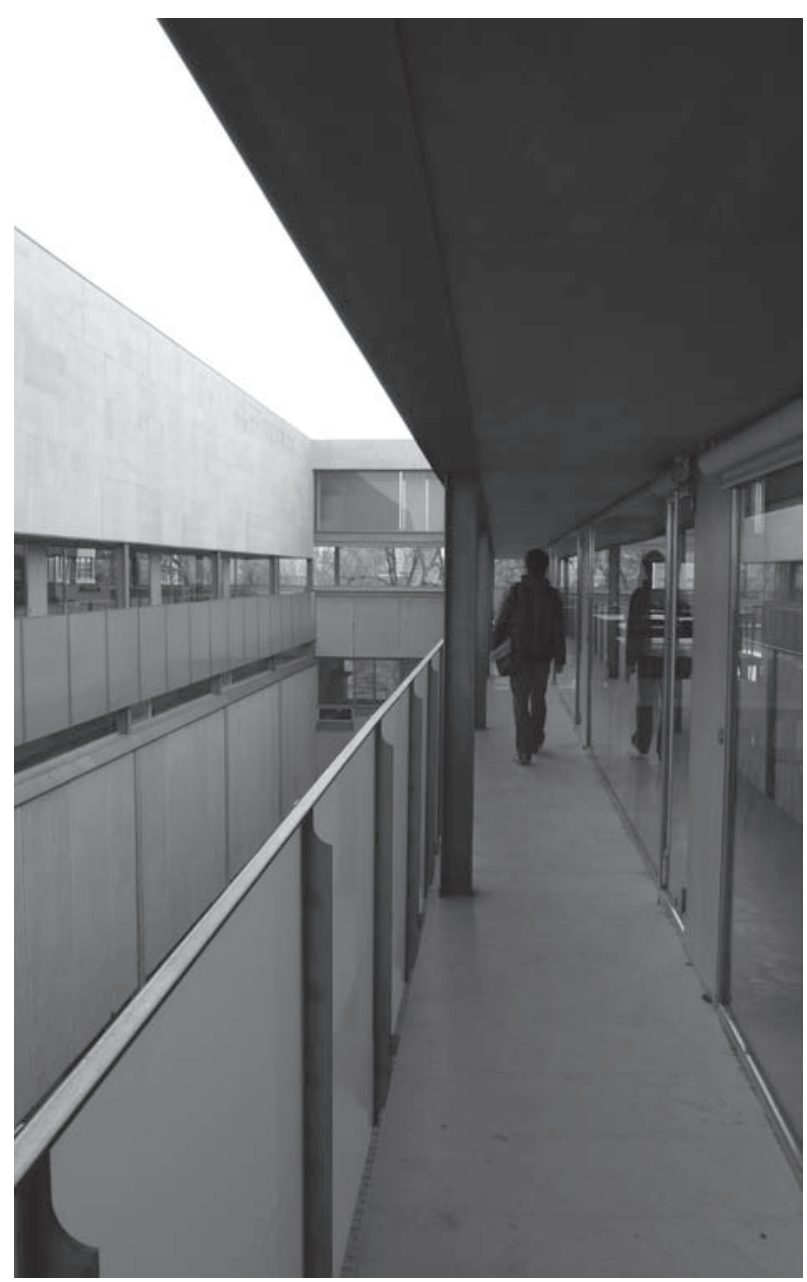

6

estudio y consulta, a cota $+4,00$, conectadas con la escuela a través de un espacio de recepción donde se sitúa el mostrador de préstamo y el acceso. (figura 7).

\section{El Aula Magna}

Desde el hall Norte se accede directamente al Aula Magna (figura 8). El aula, a la manera de las aulas académicas que Giuseppe Pagano proyecta para la universidad Bocconi, mantiene dos niveles de acceso, uno en la cota $+4,00$ y otro en la cota $+8,00$. Ambos accesos están en el eje del aula pues están vinculados al pasillo Norte, que conecta con el hall.

Este aula, de 15 × 18 × 8 m de altura, se construyó sobre un espacio vacante, que correspondía a una terraza de la planta $+4,00$. La disposición de la misma corresponde

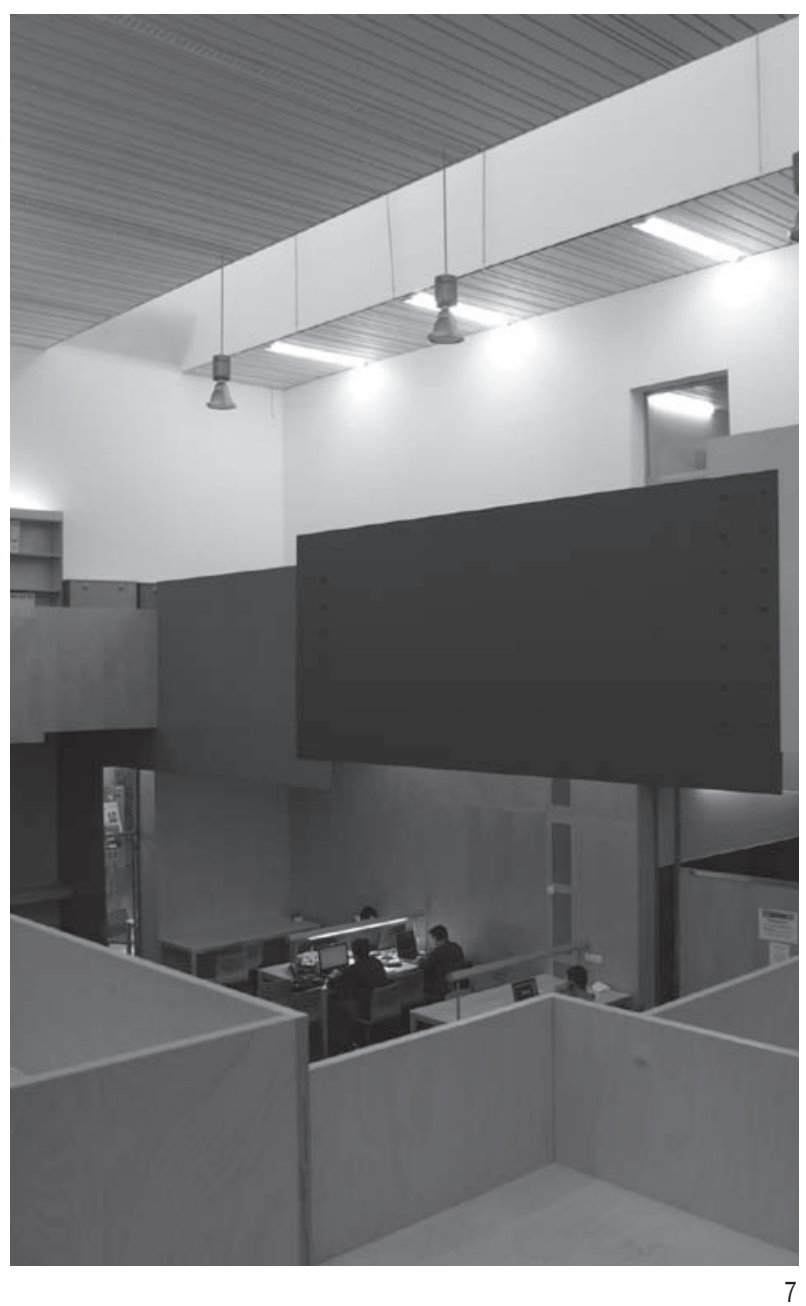

a un deseo de no perder la enfilada Este-Oeste de los recorridos internos. Para ello se introduce un gran hueco, ubicado visualmente al final del pasillo Norte, que permite a través de su transparencia introducir una cita del jardín Oeste. La versatilidad del aula exige también su oscurecimiento que se realiza a través de un panel deslizante de madera, integrado en el revestimiento.

Las butacas de la sala, en número de 344, fueron dibujadas y fabricadas especialmente para el lugar, con paleta de escritura integrada.

\section{SOBRE LA CONSTRUCCIÓN}

La estructura portante, una vez peritada, ofrecía suficiente seguridad para soportar la elevación de una planta en las zonas en la que sólo existían dos alturas. 
8. Aula Magna.

9. Fachada sur.

10. Fachada norte.

La construcción se ha resuelto mediante una estructura de acero superpuesta a la de hormigón, utilizada en forma de armazón sobre los apoyos de hormigón pertenecientes al proyecto original.

La interdependencia estructural nos permite reelaborar los códigos de pesadez, subordinándolos a la necesaria autonomía que nos asegure una operación que se muestra continua e independiente de la anterior (figura 9).

La envolvente del edificio se construye mediante un muro de dos hojas solidarias entre sí, la interior de ladrillo, y la exterior de piedra, trabadas mediante montantes de piedra de $18 \times 10 \times 90 \mathrm{~cm}$ solidarios a la fábrica de ladrillo, que permiten a través de galces laterales el ensamblaje

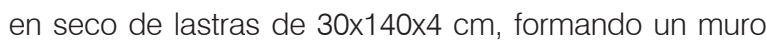
continuo terso, con aislamiento intermedio. La composición de las fachadas no responde a ninguna monótona secuencia. La regla constructiva está vinculada la optimización de cortes y ensambles (figura 10).

Esta misma voluntad compositiva se extiende a los falsos techos que introducen una vibración lineal extraída de la disposición aleatoria de cuatro anchos distintos de lamas que generan en su combinación, a la manera de un código de barras, una variación infinita. Se utilizan anchos de 3, 8,13 y $18 \mathrm{~cm}$ y longitud $6 \mathrm{~m}$ con cesuras de $2 \mathrm{~cm}$ entre ellas que absorben el ruido y se comportan como espacios moduladores del sonido (figura 5).

Los núcleos de servicio van asociados a los sistemas de circulación verticales, formados por escaleras y ascensores. Los núcleos de escaleras del proyecto preexistente nos sirven para remodelar y afirmar la posición de las nuevas escaleras. Éstas se construyen sobre la vertical de las anteriores, mediante una estructura sólida de acero sobre la que se montan los peldaños macizos y aristados de una sola pieza, de $18 \times 28 \mathrm{~cm}$, formando un paralelepípedo que mantiene la pulcritud de dos planos de distinta luz. La barandilla de acero calibrado, de $25 \mathrm{~mm}$, afirma el conjunto de la escalera con el valor de la manufactura, es decir, oficio y medida tienen una presencia independiente del diseño (figura 5).

Las zonas húmedas y los ascensores forman un frente donde la calidad del material y la presencia de planos y aristas se percibe por la costumbre más que por la excepción. Se hace compatible lo que siempre resulta marginal en el programa, como son los aseos, asumiendo una

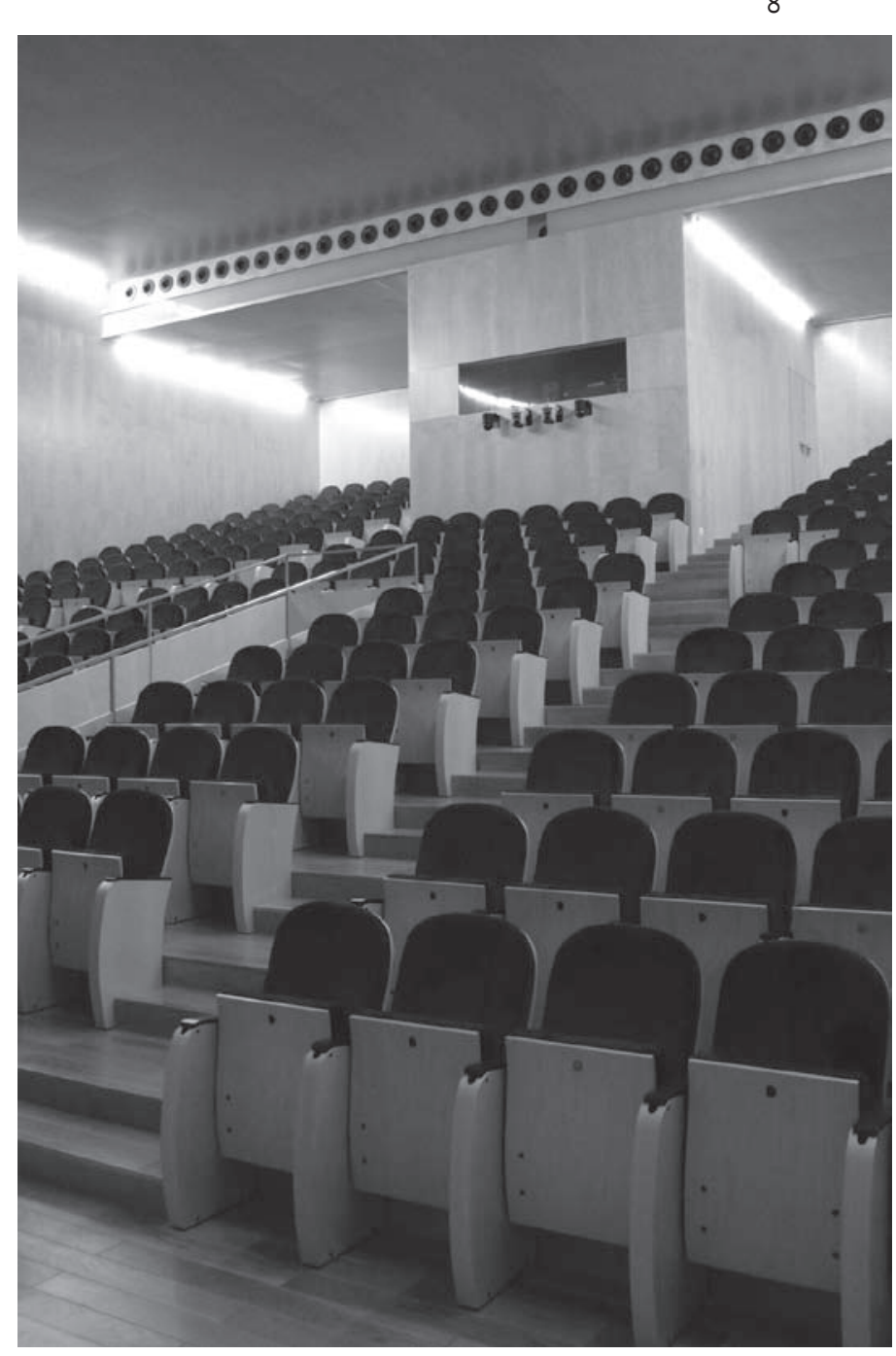

centralidad distribuida y un tratamiento de los mismos en el que se pone especial atención a un código loosiano de rigor y cuidado.

La cobertura del edificio se realiza con una plancha sándwich sustentada por la estructura metálica. El sistema de recogida de pluviales se organiza con canalones de acero inoxidable drenados en múltiples puntos por una red de tuberías horizontales asegurando 1 metro de columna de agua para garantizar la uniformidad del drenaje. Esta red se conduce a los patinillos centralizados de instalaciones permitiendo así la existencia de grandes superficies sin las interrupciones de las bajantes. 

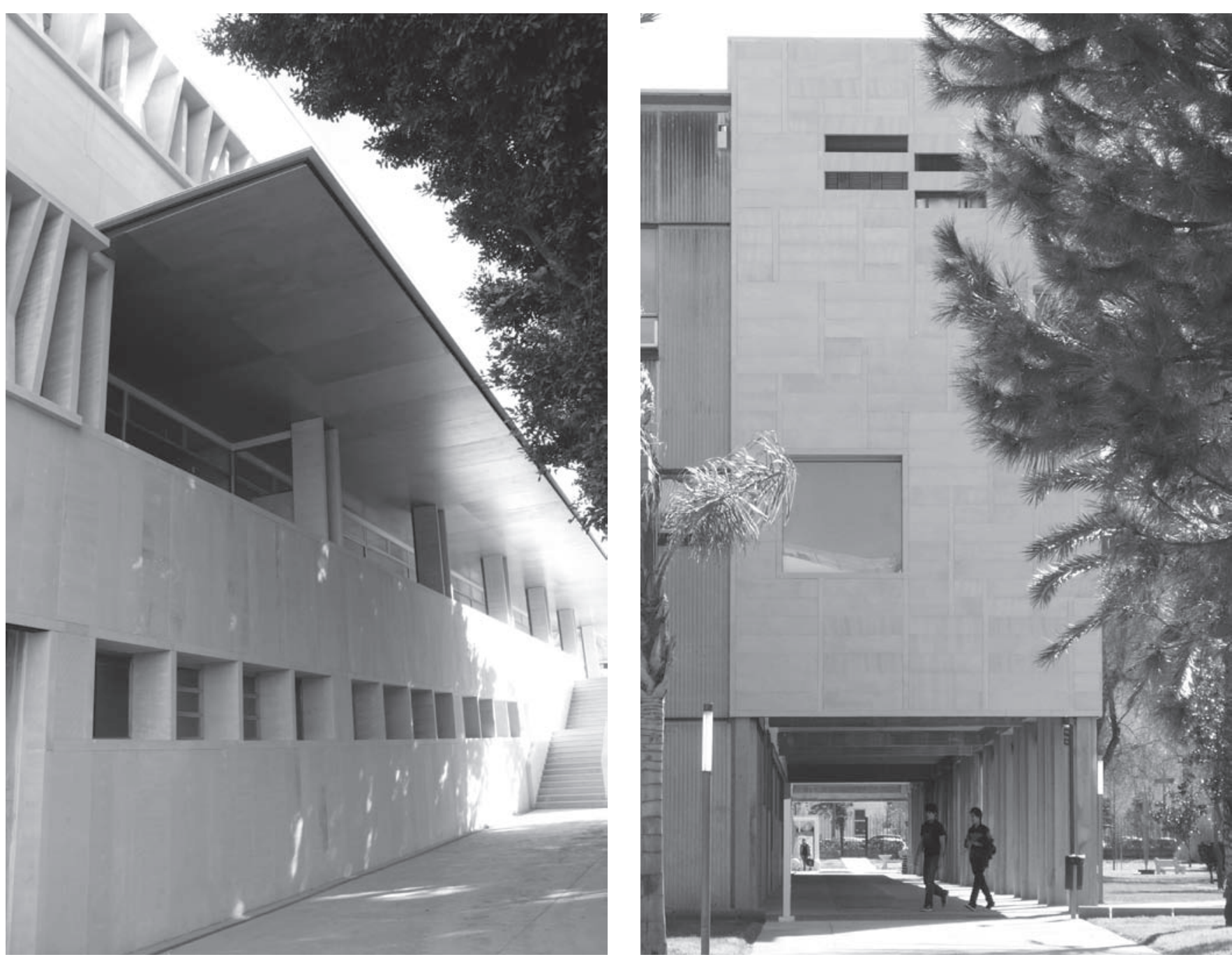

Vicente Manuel Vidal Vidal es arquitecto desde 1973 y doctor en Arquitectura desde 1982. Es profesor titular de la Escuela Técnica Superior de Arquitectura de Valencia desde 1984, catedrático del Departamento de Proyectos desde 1995. Fue Vicerrector de coordinación de Cultura y Territorio de la UPV desde 2000 a 2004, siendo actualmente coordinador en el Taller A de Proyectos Final de Carrera. En 1999 compartió un premio FAD por un proyecto de viviendas en Alcoy. Ha sido autor de proyectos de espacios públicos y de infraestructuras así como edificios de viviendas de obra nueva, administrativos, universitarios, industriales, escolares, sanitarios incluso rehabilitaciones y ampliaciones de bancos, museos y viviendas.

Ciro Manuel Vidal Climent es arquitecto desde 1997 y profesor asociado de la Escuela Técnica Superior de Arquitectura de Valencia desde el año 2005. Actualmente da clases de Proyectos I en el Taller H. Es autor de proyectos de obra nueva de edificios culturales públicos, garajes y viviendas, junto a una actividad creciente en cuanto a la rehabilitación y refuerzo estructural de conjuntos fabriles de interés cultural y edificios de viviendas en casco urbano. 